\title{
Correction to: Statistically inferred time warping: extending the cyclostationarity paradigm from regular to irregular statistical cyclicity in scientific data
}

William A. Gardner(D)

\section{Correction}

Following publication of the original article [1], the author noticed that the equation on page 9 , right column, 14th line from the bottom was incorrect. The correct equation is shown below. The original article has been corrected.

$$
\sqrt{\left(\tau_{\max }+|\tau|\right) / T} \leq \sqrt{2 \tau_{\max } / T}
$$

Received: 10 October 2018 Accepted: 10 October 2018

Published online: 25 October 2018

\section{Reference}

1. W.A. Gardner, Statistically inferred time warping: extending the cyclostationarity paradigm from regular to irregular statistical cyclicity in scientific data. EURASIP Adv. Signal Process. 2018, 59 (2018). https:/doi.org/10.1186/s13634-018-0564-6 